\title{
SOME OBSERVATIONS ON THE ASSESSMENT OF PREVENTIVE TECHNOLOGIES
}

\author{
Jeffrey P. Koplan \\ Centers for Disease Control
}

Felix F. Gutzwiller

University of Zurich

The articles in this issue of the International Journal of Technology Assessment in Health Care (IJTAHC) have explored the assessment of preventive health technologies. When considered together, these technologies provide an interesting contrast with the health care technologies that are usually evaluated on these pages. Disease prevention and its twin, health promotion, are usually practiced on a well population. Thus, many persons have the technology applied to them but only a fraction of these would have acquired the condition being prevented. Often the intervention is applied to populations rather than to individuals. The unit cost for preventive technologies is usually far less than that of diagnostic or therapeutic technologies. However, when multiplied by the larger population to be involved in the prevention program, the total costs can be considerable. In concluding this section on prevention, we would like to examine some of the larger areas of difference between preventive and other health technologies illustrated by the papers assembled here.

Screening. The early detection of disease is far more complex than merely having a screening test that is accurate, consistent, and inexpensive. Although sensitivity and specificity may be high, prevalence of the condition to be detected is an important variable because it is a critical determinant of positive predictive value. The importance we attach to false positivity and false negativity also varies by the condition. All these points are well illustrated by Rosenbrock in his consideration of HIV screening (11). The impact of a false-positive HIV test result can be psychologically devastating. A false-negative result, at a minimum, denies the person being screened information needed for planning. Even more, a false-negative result may interfere with prompt appropriate therapy and fail to motivate behaviors that will reduce risk of transmission to others. In addition, HIV screening raises the issue of the linkage between early detection and effective treatment or management. A prerequisite of a screening program is that it leads to a change in treatment and an improved outcome $(4 ; 8)$. Does a positive or negative HIV screening result alter the behavioral recommendations to avoid activities that increase risk to the person being screened or to others? Do we have effective and cost-effective treatments for which early detection of HIV positivity would be valuable? Because screening is applied to a broad population, it requires a broader 
approach than simple diagnostic testing. Ethical, legal, psychosocial, economic, and epidemiologic issues must be considered.

Assessment in Populations Rather Than Individuals. Many prevention programs are public health programs and, as such, are targeted to communities. In such programs, assessment can focus on the relative benefits, risks, and costs to the individual or to the community (10). Both analyses are useful and may yield quite different conclusions. Thus, we assess a particular vaccine for use in a given set of circumstances for an individual or look at the value of the same vaccine when used in a communitywide vaccination program. Variables such as the rates of untoward sequelae of a vaccine, the incidence of disease, who pays the cost of the vaccine and the disease, the severity and importance of a given immunizable disease for different subpopulations, the contribution of herd immunity, etc., may lead to different conclusions for the individual or for the community.

For community prevention program assessments, surveillance is a vital prerequisite (12). Surveillance of disease rates, health care practices, risk factors, or surrogates (alcohol sales) is necessary to identify problems, set policies, plan programs, and establish the baseline for subsequent evaluation efforts. Thus, in order to assess hepatitis-B immunization programs in Greece (5), we need to know the rate of hepatitis-B infection for various subpopulations. To determine the value of school health education programs, we need to know the rates of smoking and other behaviors in schoolchildren before, during, and after the intervention. In addition, as with other technologies aimed at long-term influence on behavior, such as dietary change (13) or tobacco control (7), we need to continue routine surveillance long after the termination of an intervention.

Another aspect of preventive technologies applied to a community is their relative complexity compared to diagnostic or therapeutic technologies for an individual. A community program may be more focal, such as fluoridation of the public water system (2), but frequently involves a variety of multidisciplinary activities. Thus, Chorba (1) describes using the technological devices of motor vehicle passenger restraints in the larger context of legislation, enforcement of that legislation, environmental manipulation, penalties, and public knowledge and attitudes. Similarly, Mackay and Davis (7) consider the features of successful antismoking programs and, at a minimum, list education of the public, the government, health professionals, and the media; increased taxation; curtailed advertisements; restricted smoking areas; blunt package warnings; school health education; and mass communication campaigns. Changing dietary patterns as summarized by Vartiainen et al. (13) for cardiovascular disease prevention programs requires intervening with the food industry, providing health education, addressing the accessibility of healthy dietary choices, and linking various components of a heart-healthy lifestyle. Such a multiplicity of approaches greatly complicates assessment. Attempts to isolate one component of such a program are occasionally successful, such as the ability to document the effectiveness of antismoking components of school health education (3). However, more frequently we are left with the impression that all the components work symbiotically or at least in a complementary fashion to produce the desired effect $(1 ; 7)$.

Similarly, the outcomes themselves can be difficult to measure in a community program. Process measures or intermediary outcomes may serve to substitute for "ultimate" outcomes. Thus, knowing the well-established link between smoking and lung cancer deaths, we need not wait 20 years to measure lung cancer mortality, but rather can measure smoking rates to assess our program's effectiveness. While Mackay and Davis argue that the cigarette industry's responses are a measure of an antismoking 
program's effectiveness, this form of assessment may be too indirect for many evaluators. In the case of motor vehicle collision fatalities, a health condition with a shorter latency period, prevalence of safety belt use may be a useful surveillance measure, but it can be relatively easily coupled with mortality data.

Community prevention programs are also often complicated by having more than one health outcome. Thus, antismoking programs can consider all the adverse health outcomes caused by tobacco: lung cancer, oral cancer, coronary heart disease, chronic obstructive lung disease, etc. In contrast, what is arguably one of the most effective and cost-effective prevention programs, community water fluoridation, has relatively easily measurable outcomes: decayed, missing, or filled teeth, the DMF index (2).

Technology Assessment and Public Policy. Because public health programs involve an explicit expenditure of public funds, community prevention efforts have long been held up to scrutiny and evaluated in a way that therapeutic interventions have not. Thus, there is a long history of drugs, devices, and surgical procedures being widely implemented with little assessment, and then restricted over time as assessments are performed. Prevention technologies are judged for safety, effectiveness, and costeffectiveness early in their development. Vaccine programs illustrate such a logical process, as seen in the stepwise increases in hepatitis-B vaccine use worldwide. HIV screening, injury control, fluoridation, school health education, and smoking prevention programs have all been the subject of public debate and professional study, with the subsequent consideration of the broad range of concerns that make up technology assessment. Clinical health technologies rarely are subjected to such scrutiny - be they electronic fetal monitoring, surgery for coronary heart disease, or hysterectomy $(6 ; 9)$.

Preventive approaches are usually preferable to the clinical alternatives, both conceptually and in terms of consumer satisfaction. This is not to say that all preventive approaches are effective, or cost-saving, or even cost-effective relative to other approaches. Nevertheless, preventive technologies offer much as a way to avert suffering, disability, death, and health care costs. Health care professionals interested in technology assessment should apply their skills to health promotion and disease prevention technologies to more widely diffuse those that are found to be effective, to limit those that are not, and to better define the circumstances under which the technologies should be used.

\section{REFERENCES}

1. Chorba, T. L. Assessing technologies for preventing injuries in motor vehicle crashes. International Journal of Technology Assessment in Health Care, 1991, 7, 296-314.

2. Corbin, S. B. Oral disease prevention technologies for community use. International Journal of Technology Assessment in Health Care, 1991, 7, 327-44.

3. Dwyer, T., Viney, R., \& Jones, M. Assessing school health education programs. International Journal of Technology Assessment in Health Care, 1991, 7, 286-95.

4. Eddy, D. M. Screening for cancer. Englewood Cliffs, NJ: Prentice Hall, 1980.

5. Hatziandreu, E. J., Hatzakis, A., Hatziyannis, S., Kane, M. A., \& Weinstein, M. C. Costeffectiveness of hepatitis-B vaccine in Greece: A country of intermediate HBV endemicity. International Journal of Technology Assessment in Health Care, 1991, 7, 256-62.

6. Institute of Medicine. Assessing medical technologies. Washington, DC: National Academy Press, 1985.

7. Mackay, J., \& Davis, R. M. Assessing community interventions to reduce smoking. International Journal of Technology Assessment in Health Care, 1991, 7, 345-53.

8. Morrison, A. S. Screening in chronic disease. New York: Oxford University Press, 1985.

9. Office of Technology Assessment. Assessing the efficacy and safety of medical technologies. Washington, DC: U.S. Government Printing Office, 1978. 


\section{Koplan and Gutzwiller}

10. Redelmeier, D. A., \& Tversky, A. Discrepancy between medical decisions for individual patients and for groups. New England Journal of Medicine, 1990, 322, 1162-64.

11. Rosenbrock, R. D. Screening for human immunodeficiency virus. International Journal of Technology Assessment in Health Care, 1991, 7, 263-74.

12. Thacker, S. B., \& Berkelman, R. L. Surveillance of medical technologies. Journal of Public Health Policy, 1986, 7, 363-77.

13. Vartiainen, E., Heath, G., \& Ford, E. Assessing population-based programs to reduce blood cholesterol level and saturated fats. International Journal of Technology Assessment in Health Care, 1991, 7, 315-26. 